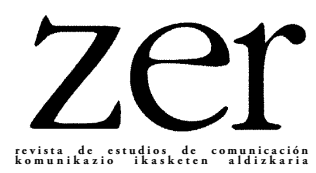

\title{
Pautas de redacción y análisis de contenido en noticias sobre suicidio en la prensa española e internacional: efecto Werther\&Papageno y seguimiento de las recomendaciones de la OMS
}

Espainiako eta nazioarteko prentsan suizidioari buruzko berrien edukia idazteko eta aztertzeko jarraibideak: WertherEPapageno efektua eta OMEren aholkuen jarraipena

Guidelines and analysis of the content of news about suicide in Spanish and international press: Papageno and Werther effect and level of compliance of WHO advice

\author{
Melani Lois-Barcia`, Icía Rodríguez-Arias, Miguel Túñez \\ Universidad de Santiago de Compostela
}

\begin{abstract}
RESUMEN: En España fallecen por suicidio el triple de personas que en accidente de tráfico, pero la muerte intencional sigue siendo un asunto que en las redacciones informativas se silencia por considerar que su visibilidad fomenta el aumento de conductas imitativas. La Organización Mundial de la Salud promueve desde 2000 una guideline para el abordaje informativo del suicidio en la que se proponen comportamientos diferentes: informar con responsabilidad y de modo que se colabore en dinámicas de prevención. Esta investigación revisa libros de estilo o códigos de conducta de FAPE, de los nueve colegios profesionales de España y de 23 organizaciones profesionales internacionales y analiza las noticias online durante seis meses en una muestra intencional de 11 periódicos de referencia (El País, El Mundo, La Vanguardia, La Voz de Galicia $y$ El Periódico, The Guardian, Le Monde, The New York Times, The Washington Post, Corrieri della Sera y Público) para identificar si existen pautas que orienten el abordaje informativo e intentar determinar si la política informativa apuesta por fomentar el efecto Werther (silencio) o el efecto Papageno (prevención).
\end{abstract}

PALABRAS CLAVE: Medios de comunicación, responsabilidad social, prensa, suicidio.

LABURPENA: Espainian, suizidioa dela eta, trafiko istripuetan hiltzen diren pertsonen hirukoitza hiltzen da. Hala ere, nahitako heriotza informazio erredakzioetan isila- 
razten den gaia da oraindik ere, horren ikusgaitasunak imitazio jokabideak sustatuko dituelakoan. Osasunaren Mundu Erakundeak, 2000. urtetik, guideline bat proposatzen $d u$ suizidioa informazioaren alorrean artatzeko; bertan, jarrera ezberdinak proposatzen dira: arduraz eta prebentzio dinamikak sustatzeko moduan informatzea. Ikerketa honetan, FAPEren estilo liburuak edo jokabide kodeak aztertzen dira, Espainiako bederatzi elkargo profesionalenak eta nazioarteko hogeita hiru elkarte profesionalenak. Horrez gain, sei hilabetean zehar, erreferentziazko 11 egunkariren online berriak ere aztertzen dira (El País, El Mundo, La Vanguardia, La Voz de Galicia eta El Periódico, The Guardian, Le Monde, The New York Times, The Washington Post, Corrieri della Sera eta Público), informazioa artatzera bideratutako jarraibideak dauden ikusteko eta horiek identifikatzeko, eta informazio politikak Werther efektuaren (isiltasuna) edo $\mathrm{Pa}-$ pageno efektuaren (prebentzioa) alde egiten duen zehazten saiatzeko.

HITZ-GAKOAK: Komunikabideak, erantzukizun soziala, prentsa, suizidioa.

\begin{abstract}
In Spain three people commit suicide for each traffic accident but intentional death is still silenced in newspapers for understanding that its visibility promotes imitation of this kind of behavior. The World Health Organization encourages since the year 2000 a guideline for suicide coverage in which different conducts are included; to inform responsibly and aiming prevention dynamics. This investigation looks through style guides and codes of ethics from the FAPE, Spanish Journalism associations and 23 international professional organizations. It analyzes online news from a six-month period in several media (El Pais, El Mundo, La Vanguardia, La Voz de Galicia y El Periódico, The Guardian, Le Monde, The New York Times, The Washington Post, Corrieri della Sera y Público) to identify if there is any guidance that orders the informative coverage. This study has also the aim to find out if these neewspapers tend to follow Werther (silence) or Papageno (prevention) effect.
\end{abstract}

KEYWORDS: Media, social responsability, press, suicide.

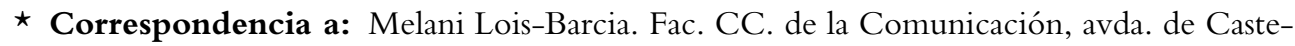
lao, s/n. Campus norte (15782 Santiago de Compostela).melani.lois@rai.usc.es http://orcid.org/0000-0001-5785-8620

Cómo citar: Lois-Barcia, Melani; Rodríguez-Arias, Icía; Túñez, Miguel. 2018. Pautas de redacción y análisis de contenido en noticias sobre suicidio en la prensa española e internacional: efecto Werther\&Papageno y seguimiento de las recomendaciones de la OMS. Zer. 23(45). 139-159.

https://doi.org/10.1387/zer.20244

Recibido: 16 setiembre 2018; aceptado: 18 octubre 2018

1137-1102 y 1989-631X / (C) 2018 UPV/EHU

(c) (1) Esta obra está bajo una licencia Creative Commons Atribución 4.0 Internacional 


\section{Werther o Pagageno, silencio o prevención como actitud informativa}

En el mundo se suicidan 800.000 personas cada año y por cada caso se producen varias tentativas. Igual que en las noticias sobre violencia de género y accidentes de tráfico, la manera que tenemos de contar las cosas puede cambiar la realidad. En esta cuestión es vital. La manera en la que nos referimos a una persona que cometió el acto suicida puede ser clave para evitar futuras pérdidas. En palabras del presidente de la Sociedad Española de Suicidiología, Andoni Anseán, en una entrevista de la agencia EFE: «en España y en otros países del entorno, los hombres jóvenes se mueren por suicidio, pero ese dato no sale en los medios de comunicación y es sorprendente: nadie quiere ver el elefante que tenemos en la habitación».

Informar sobre suicidio es una compleja tarea que en las redacciones se aborda mayoritariamente desde la creencia de que es un tema a excluir del temario porque la notoriedad del acto actúa de estímulo para desencadenar situaciones similares en otros individuos. En España, los últimos datos oficiales indican que el número de suicidios (ausentes de temario) triplican al número de muertes en accidentes de tráfico (presentes cada día en los medios). Sobre el suicidio hay, "una regla no escrita, a modo de autocensura, que dicta que no se debe informar de ellos, salvo en casos excepcionales, para evitar un efecto imitación entre los miembros vulnerables del público o la audiencia» (Olmo y García, 2015: 71).

Los estudios de expertos en Comunicación sobre la presencia de noticias en los medios de masas o sobre el modo de elaborar el contenido informativo son escasos. Los análisis acostumbran a realizarse desde las áreas de salud, principalmente psiquiatría y psicología, pero no tanto desde la perspectiva de expertos en newsmaking o en agenda setting que puedan analizar en profundidad los mecanismos que se activan o se detienen para determinar la selección de noticias sobre suicidio, el modo de presentarlas o descartarlas en la construcción del temario, su ubicación en secciones de salud o de sucesos, la selección de datos para el relato noticioso y el modo de jerarquizarlos y de abordar la narración final del hecho. Los enfoques de los trabajos científicos acostumbran a orientarse a los efectos sobre las audiencias y a valorar el impacto de los efectos Werther y Papageno en función de la intencionalidad de la información y de sus posibles efectos sobre la audiencia.

Sobre el suicidio como acontecimiento informativo los medios han adoptado un rol protector y han optado por silenciar aliándose con lo que el sociólogo David Philips denominó en 1974 como el efecto Werther, en alusión a lo acontecido a finales del siglo XVII cuando en países como Italia o Dinamarca se prohibió la venta de Las penas del joven Werther, de Johann Wolfgang von Goethe, porque tras su publicación en 1774 se detectaron casos de jóvenes que imitaban al protagonista suicida. La relación entre la notoriedad del sujeto y el impacto imitativo en la población, es una 
línea que sustentan, entre otros, los análisis de Philips, Lesyna e Paight (1992), Wasserman (1984) y Müller (2011) aunque también se cuestiona si el impacto es por la información sobre el hecho en sí o sobre el modo de narrar el acontecimiento, en ocasiones con sesgos literarios y erróneas asociaciones que conviertan la noticia en un texto sensacionalista o adornada con tintes románticos.

Derivar el suicidio a la realidad silenciada por los medios puede provocar, también, efectos que inciden negativamente sobre la población. La antítesis del efecto Werther está en el efecto Papageno según el cual la cobertura responsable de una noticia puede tener un efecto preventivo sobre la conducta suicida. Los defensores del efecto Papageno proponen que se informe de casos en los que se logró superar episodios de crisis suicida porque de ese modo el efecto imitativo podría incidir beneficiosamente sobre la población. «El impacto de la información sobre el suicidio no puede limitarse a los efectos nocivos, sino que la cobertura del abordaje positivo en circunstancias adversas, como se explica en los artículos de prensa sobre la ideación suicida, puede tener efectos protectores» (Niederkrotenthaler, 2012, en Herrera, 2015).

En general, en la literatura científica se apuesta por un equilibrio de ambos efectos. Olmo y García sostienen que "para eso se requiere informar responsablemente, sin sensacionalismos, pero sin eufemismos y tratándolo como un tema de salud pública» (2015: 88) y defienden que sean las propias organizaciones profesionales de periodistas las que, con asesoramiento de expertos en salud, generen sus códigos de actuación. Garrido-Fabian et al., por su parte, aseguran que en las investigaciones sobre ambos efectos concluyen que el impacto mediático de los suicidios es un continuo entre ambos efectos (2012: 812) y demandan «más investigación para evaluar la calidad del tratamiento de los medios de comunicación de sobre suicidios (2018: 821).

Entre las escasas propuestas localizadas en trabajos del área de Comunicación destacan el análisis del efecto Werther y del modo de informar de influencia de suicidios de personajes conocidos y su impacto en los receptores (Müller, 2011) o del efecto Papageno en la prensa española (Herrera, Ures y Martínez, 2015). La revisión de revistas científicas del área revela, también, las investigaciones de Olmo y García con mirada al pero de la información televisiva (2015) o su análisis sobre la relevancia pública y la responsabilidad social del tema (2014), y de Garrido-Fabián, Serrano-López y Catalán-Matamoros (2018), que estudian el uso por los periodistas de las recomendaciones de la OMS a través del análisis del diario ABC entre 1995 y 2005, periodo en el que detecta una tendencia "a reducir el número de noticias de suicidios específicos que incluyen descripciones del método empleado y la publicación de notas de suicidio, que presentan el suicidio como inexplicable o causado por un sólo factor y que lo describen como método para enfrentar problemas en mayor medida» (2018: 821).

Otros estudios científicos desde Comunicación abordan el tratamiento periodístico en la prensa canaria a finales de la primera década de este siglo (Carrascosa, 
2015), la información sobre suicidio en Chile (Urzúa, 2003) o la caracterización de noticias sobre suicidio en Colombia (Muñoz, 2014 y Rátiva, Ruíz y Medina-Pérez, 2013) o sobre el abordaje de medios colombianos y de manuales de estilo de periódicos de Colombia y España (Rubiano, Quintero y Bonilla, 2007). A la lista se suman referencias de trabajos en el entorno europeo, como la revisión de diarios Suecos (Catalán-Matamoros, Axelsson y Strid, 2007) y británicos (Goulden et al, 2011, Thornicroft et al, 2013) o las referenciales aportaciones de Philips (1982 y 1992), Norris (2001), Stack (2003), Niederkrotenthaler et al (2010) o Sisask y Värnik (2012), Ladwig el al (2012) o Scherr y Steinleitner, (2015).

Olmo y García $(2015,77-78)$ aportan un detallado recorrido por iniciativas en torno a un buen abordaje informativo del suicidio en el que incluyen la revisión crítica del trabajo realizado sobre el papel de los medios en la prevención elaborada por Sisask y Värnik (2012), los informes y normas para hablar sobre suicidios promovidos por la ONG británica Media Wise Trust, integrada por periodistas preocupados en fomentar prácticas honestas y éticas, y el trabajo de Pirkis y Blood (2010) en favor de que los medios contribuyan a la prevención. También refieren las recomendaciones del gobierno Australiano y su política intervencionista en la orientación de los contenidos mediáticos, la guía The reporting of Mental Health and Suicide by the Media publicada en 2006 por la National Union of Journalists of Scotland, a la propia Media Wise Trust y a la ONG Samaritans que se cita como referente para ampliar información sobre tratamiento correcto en el libro de estilo de la BBC, que dedica uno los capítulos de sus guidelines a cómo tratar «suicidio, intento de suicidio, autolesión y desórdenes de la conducta alimentaria» (Olmo y García, 2015, 85-86).

\subsection{LAS RECOMENDACIONES DE LA OMS}

El punto de equidistancia entre ambos efectos e intencionalidades informativas podría situarse en las recomendaciones de la Organización Mundial de la Salud que en un documento instructor para profesionales de los medios de comunicación resalta que se debe «informar del suicidio de manera adecuada, exacta y potencialmente útil a través de medios progresistas e inteligentes puede prevenir una trágica pérdida de vidas» (OMS, 2000).

Según la OMS, suicidio es un acto deliberadamente iniciado y llevado a cabo por un individuo con el conocimiento o la expectativa de que su resultado va a ser la muerte que se sitúa entre las diez primeras causas de muerte en el mundo (en torno a un millón de muertes al año). La magnitud del problema crece ya que se estima que hay un fenómeno iceberg: se estima que por cada suicidio consumado existen al menos 20 intentos pero se dispone a nivel mundial registros oficiales de comportamientos de riesgo suicida y la mayoría de intentos no se informan ni se registran. 
Las cifras sobre causas de la muerte que ofrece el Instituto Nacional de Estadísticas (INE) en 2108 registran 3.569 suicidios en España. Por género, la tasa de hombres (2.662) triplica la de mujeres (907). Los datos recogen oficialmente las defunciones de 2016 (se publican siempre dos años más tarde) y sitúan el suicido por delante de fallecimientos por paro cardíaco (3.460) y en accidentes de tráfico (1.890). Los casos se reparten entre 1.653 episodios por ahorcamiento, estrangulamiento o sofocación autoinflingida y 904 por salto intencionado desde un lugar elevado. Por comunidades, en términos absolutos el mayor número se concentra en Andalucía seguida de Cataluña, Galicia y Comunidad Valenciana, aunque al ponderar la incidencia en función del número de habitantes la lista se invierte y Galicia lidera con diferencia $(124,31$ casos por millón de habitantes). La falta de visibilidad mediática del suicidio hace que también resulte sorprendente analizar la pirámide de edad: el pico de incidencia está en la población de 30 a 39 años y, en general, a medida que la edad aumenta el número de suicidios desciende, aunque su incidencia es trasversal a toda la pirámide de edad.

Las propuestas de la Organización Mundial de la Salud son punto de partida para este trabajo sintetizando en su ficha de análisis las recomendaciones de la OMS para la correcta orientación de informaciones sobre actos suicidios incorporada al programa SUPRE (SUicide PREvention) como uno de los instrumentos dirigidos a colectivos profesionales que puedan ser «particularmente relevantes» en la prevención del suicidio, entre ellos los «comunicadores».

El documento de la OMS (2000) (http://www.who.int/mental_health/ publications/suicide_prevention/es/index) remite a las web de la Asociación Internacional para la Prevención del Suicidio, la Asociación Americana de Suicidología, la Red Australiana de Intervención Temprana para Salud Mental en Personas Jóvenes y la Academia Internacional de Investigación del Suicidio para ver las guías profesionales que han elaborado, pero añade su propia guideline en la que se deja libertad narrativa al periodista y se recomienda que en informaciones generales sobre el tema se interpreten las estadísticas cuidadosamente, se usen fuentes auténticas y confiables, se manejen con cuidado comentarios espontáneos, se eviten las magnificación de pequeñas cifras con expresiones superlativas y se aluda identificar el comportamiento suicida "como una respuesta entendible a los cambios o la degradación social o cultural» $(2000,9)$.

En las noticias sobre un suicidio concreto, se pide evitar: una cobertura sensacionalista, sobre todo si involucra a un actor social relevante; las fotografias de la víctima o de la escena del suicidio; y «las descripciones detalladas del método usado y cómo lo obtuvo la víctima» (2000: 9). También incide en que no se presente el suicidio como «algo inexplicable o simplista» porque «nunca es el resultado de un solo factor o hecho» sino que está causado por «una compleja interacción de muchos factores tales como enfermedad mental y fisica, abuso de sustancias, conflictos familiares e interpersonales y acontecimientos estresantes» (OMS, 2000: 9-10). 
El relato informativo, según la propuesta de la OMS, debe alejarse de describir al suicidio como un método de enfrentarse a problemas personales/sociales, debe orientarse a lamentar la muerte más que a presentar a la víctima como mártir o con términos de adulación, y a tener en cuenta el impacto sobre las familias y otros sobrevivientes en términos del estigma y el sufrimiento psicológico. Además, se anima a "describir las consecuencias físicas de intentos de suicidio (daño cerebral, parálisis, etc.)» porque "puede actuar como elemento de disuasión» $(2000,10)$.

La guía anima a los medios de comunicación a comportarse como actores proactivos en la prevención del suicidio «contextualizando las informaciones con listados de servicios de salud mental y líneas telefónicas de ayuda disponibles con sus números y direcciones actualizados, publicitar las señales de advertencia del comportamiento suicida, transmitir mensajes sobre la frecuente asociación entre la depresión y el comportamiento suicida, ofrecer un mensaje de solidaridad a los sobrevivientes» (2000: 10).

A modo de resumen sintetiza sus recomendaciones en pautas de comportamiento con lo que se debe hacer: trabajar estrechamente con autoridades de la salud en la presentación de los hechos; referirse al suicidio como un hecho logrado, no uno exitoso; presentar sólo datos relevantes en las páginas interiores; resaltar las alternativas al suicidio; proporcionar información sobre líneas de ayuda y recursos comunitarios; publicitar indicadores de riesgo y señales de advertencia (OMS, 2000: 11) y lo que se debe evitar: No publicar fotografias o notas suicidas; no informar detalles específicos del método usado; no dar razones simplistas; no glorificar ni magnificar el suicidio, no usar estereotipos religiosos o culturales; no aportar culpas (OMS, 2000: 11).

\section{Metodología}

Para analizar el abordaje informativo del suicidio se diseñó una investigación exploratoria con metodología triangular en las que, además de revisar pormenorizadamente el estado de la cuestión, se orientó a conocer las recomendaciones, pautas o normas de los principales periódicos y de las organizaciones profesionales más representativas y se realizó un análisis de contenido en informaciones publicadas para determinar si el enfoque de la narración en informaciones sobre suicidio se ajusta a las recomendaciones de la Organización Mundial de la Salud.

El principal objetivo es conocer si en las informaciones sobre suicidio se asumen las recomendaciones de la OMS y, por extensión, si se alinean en el efecto Werther (presencia escasa y centrada en personajes notarios) o en el efecto Papageno (incorporando referencias adicionales orientadas a prevención y apoyo). Además, se formularon de partida los siguientes objetivos/preguntas de investigación: ¿los me- 
dios incluyen el tratamiento del suicidio en sus libros de estilo?, ¿las organizaciones profesionales incluyen el suicidio en sus normas/recomendaciones?, ¿hay diferencias entre los medios nacionales y los internacionales?, ¿hay diferencias entre las organizaciones españolas y las de otros países?

Para identificar la existencia de normas o recomendaciones para elaborar informaciones sobre un suicidio se, en España se trabajó con la página web de la FAPE y las de los nueve Colegios Profesionales de Periodistas registrados en España pertenecientes a Andalucía, Asturias, Castilla y León, Cataluña, Euskadi, Galicia, La Rioja, Murcia y Navarra.

En el ámbito internacional se seleccionaron como sujetos de estudio 23 organizaciones: Centre for Investigative Journalism, Deutscher Journalisten-Verband, Fourth Estate (association), IFEX, Inter American Press Association, International Association of Press Clubs, International Center for Journalists, International Council for Press and Broadcasting, International Federation of Agricultural Journalists, International Francophone Press Union, International Journalism Festival, International Media Support, International Press Center (Brussels), International Press Institute, International Women's Media Foundation, Internationales Institut für Journalismus, Media Legal Defence Initiative, Mideast Dig, Technical Image Press Association, World Press Institute, Youth Journalism International, Internews y Media Development Investment Fund.

No se encontraron investigaciones anteriores en el área de Comunicación con un abordaje múltiple y comparativo de códigos de medios y entidades profesionales, y de estudio global (nacional e internacional) del seguimiento de las recomendaciones de la OMS en las informaciones sobre suicidio. Por eso, para el análisis de medios se estableció una muestra intencional basada en los cinco diarios nacionales generalistas mejor ubicados en términos de audiencia en el EGM (febrero-noviembre, 2017) y un corpus internacional compuesto por seis medios de referencia de Europa y Estados Unidos que sirve de elemento de contraste.

La muestra de diarios nacionales quedó confirmada por El País, El Mundo, La Vanguardia, La Voz de Galicia y El Periódico. La muestra de periódicos internacionales la integran The Guardian (Uk), Le Monde (Fr), The New York Times (EEUU), The Washington Post (EEUU), Il Corriere della Sera (It) y Público (Pt). Se analizó la versión online de las 11 cabeceras mediante búsquedas en Google para evitar distorsiones de los buscadores de cada medio. El periodo seleccionado fue de abril a septiembre, los seis meses con mayor número de suicidios según el INE. Se afinó la búsqueda para que los resultados se limitasen a informaciones del diario buscado colocando en el buscador insite:webdelmedio suicidio (por ejemplo, insite:elpais.com suicidio).

Los resultados de cada medio se filtraron siguiendo los siguientes requisitos: tratar el suicidio de una persona como hecho principal, siendo este lo más importante 
dentro de la pieza, o, al menos, de la misma importancia que otros acontecimientos descritos; no se recogen los ataques suicidas ni los intentos de suicidio; solo se incluirán géneros informativos; no se seleccionan informaciones sobre hechos supuestos en las que no se conozca realmente que es un caso de suicidio y quedan fuera de la selección piezas periodísticas limitadas a resúmenes estadísticos. Tras el filtrado, los 2.127 enlaces iniciales quedaron reducidos a 258 eliminando también entradas duplicadas y obteniendo la muestra de análisis de noticias final.

Para determinar el grado de seguimiento de las recomendaciones de la OMS se determinó una ficha de análisis en la que se anota fecha de análisis de la noticia, medio y se verifica si:

- Proporciona información sobre líneas de ayuda y recursos comunitarios. Se requiere que en la noticia aparezca algún dato de contacto de entidades que puedan servir como un primer referente a alguien en riesgo de suicidio, o aportar información a familiares, amigos...

- Proporciona indicadores de riesgo y señales de advertencia. Se considera que siguen esta recomendación los textos que expliquen algún comportamiento que pueda ayudar a detectar cuando alguien necesita ayuda. Es decir, aspectos que puedan interpretarse como indicadores para identificar esas llamadas de auxilio.

- Publica fotografías o notas suicidas. Imágenes de la persona en los momentos previos, durante o después del suicidio. En el caso de las notas suicidas, se contabiliza tanto si se ofrece escaneada, fotografiada o reproducida en el texto.

- Aporta detalles específicos del método empleado. Se considera que los aporta si va más allá de identificar el modo del fallecimiento y entra en relato del mismo.

- Aporta razones simplistas. Si en el texto hay conjeturas del autor sobre los motivos sin profundizar o si hay frases que pretenden justificar la acción.

- Aporta culpas. Si señala a personas físicas o jurídicas como la causa de la muerte porque se aleja de considerar el suicidio como una acción emprendida por una persona ante la situación en la que vive, generalmente experimentando incapacidad para gestionarla.

- Resalta alternativas al suicidio o aporta pautas que pudieran llevar a alguien con tendencia suicida a buscar apoyo para paliar su sufrimiento y superar la situación adversa.

Las noticias analizadas corresponden a los meses comprendidos entre el 1 de abril de 2017 hasta el 30 de septiembre de 2017. La búsqueda, selección y análisis se realizaron de febrero a abril de 2018. La interpretación y valoración de los resultados se llevó a cabo en mayo. 


\section{Resultados}

Para aproximarnos a conocer qué medios tienen una preocupación real por abordar el suicidio de una forma correcta, cuáles sólo se limitan a recoger éticamente el tema y cuáles lo omiten tanto a nivel ético-teórico como a nivel práctico estudiamos en primer lugar las referencias al abordaje informativo del suicidio en los documentos deontológicos o de estilo de los medios nacionales e internacionales escogidos y en las organizaciones profesionales de referencia de nuestro país y en el ámbito internacional.

\subsection{AnÁlisis De Códigos deONTOlógicos y libros De ESTILO}

TABLA 1

Análisis de códigos deontológicos/libros de estilo de los medios nacionales

\begin{tabular}{|l|l|l|l|}
\hline \multicolumn{1}{|c|}{ Medio } & \multicolumn{1}{|c|}{ URL } & \multicolumn{1}{c|}{$\begin{array}{c}\text { Incluye } \\
\text { recomendaciones para } \\
\text { informar sobre suicidio }\end{array}$} & $\begin{array}{c}\text { Acorde a las pautas } \\
\text { de la OMS }\end{array}$ \\
\hline El Mundo & www.elmundo.es & Sí. & Sí. \\
\hline La Vanguardia & www.lavanguardia.com & No. \\
\hline La Voz de Galicia & www.lavozdegalicia.es & No. & No. \\
\hline El Periódico & www.elperiodico.com & No. \\
\hline El País & www.elpais.com & Sí. & No. \\
\hline
\end{tabular}

Fuente y elaboración propias.

De los cinco periódicos nacionales con mayor número de lectores según el EGM (Estudio General de Medios), sólo El Mundo y El País proporcionan contenido orientativo sobre el suicidio en sus códigos éticos. El Mundo resalta que se trata de una cuestión delicada e invita al periodista a reflexionar sobre dos cuestiones: el efecto Werther y la confirmación de que se trata de un suicidio, para evitar por una parte el efecto de imitación y por otra la difusión de bulos. Incide en indicar si existen hipótesis que apuntan al suicidio, pero no confirmarlo si las autoridades y expertos aún no lo hicieron. Añade, además de un ejemplo para mostrar que se puede llegar a transgredir algún derecho personal si no se hace un buen tratamiento del suicidio, una parte jurídica que va más allá de la autorregulación periodística.

El País muestra interés, pero no desempeña un papel activo en la prevención ya que únicamente señala que debe ser tratado con cautela y que sólo deben 
cubrirse suicidios de personajes relevantes o si supone un hecho social de interés general. En su Libro de Estilo se refiere de modo conciso al suicidio en la sección de Política Editorial de su Título I. se aboga por la prudencia porque «la psicología ha comprobado que estas noticias incitan a quitarse la vida a personas que ya eran propensas al suicidio y que sienten en ese momento un estímulo de imitación».

TABLA 2

Análisis de códigos deontológicos/libros de estilo de los medios internacionales

\begin{tabular}{|l|l|l|l|}
\hline \multicolumn{1}{|c|}{ Medio } & \multicolumn{1}{|c|}{ URL } & \multicolumn{1}{c|}{$\begin{array}{c}\text { Incluye } \\
\text { recomendaciones para } \\
\text { informar sobre suicidio }\end{array}$} & $\begin{array}{c}\text { Acorde a las pautas } \\
\text { de las OMS }\end{array}$ \\
\hline The Guardian & theguardian.com & Sí. & Sí. \\
\hline The New York Times & nytimes.com & No. \\
\hline The Washington Post & washingtonpost.com & No. & No. \\
\hline Público (Pt) & www.publico.pt & No. \\
\hline Corriere della Sera & www.corriere.it & No. & No. \\
\hline Le Monde & www.lemonde.fr & No. No. \\
\hline
\end{tabular}

Fuente y elaboración propias.

En esta muestra integrada por medios de referencia a nivel internacional se percibe la carencia de interés por parte de los profesionales de la comunicación a la hora de abordar el suicidio. Sólo el periódico The Guardian, incluye una serie de recomendaciones bastante completas además de estar en sincronía con las de la OMS. Comienza invitando a tener cuidado con la cobertura de los suicidios, pero va más allá al especificar que no se debe profundizar en el método de suicidio empleado. Hace hincapié, además, en tener cuidado con los sentimientos de los familiares y en tratar de escribir mostrando empatía por los mismos. Pero lo más útil de lo que recoge para la prevención del suicidio, es que recomienda adjuntar el número de teléfono de una línea de ayuda tal y como defiende la OMS.

\subsection{Análisis de Colegios Profesionales, FAPE y organizaciones INTERNACIONALES}

La Federación de Asociaciones de Periodistas de España (FAPE), entidad de referencia del asociacionismo profesional a nivel nacional no posee recomendaciones o pautas específicas para un correcto tratamiento del suicidio en su código deontoló- 
gico, pero sí que contiene fragmentos que podrían ser aplicados a este tema. En su artículo 4, por ejemplo, se potencia el respeto al derecho de las personas a su propia intimidad e imagen justificando las intromisiones o indagaciones sobre la vida privada de una persona sin su previo consentimiento sólo en caso de interés público. Señala también que «en el tratamiento informativo de los asuntos en que medien elementos de dolor o aflicción en las personas afectadas, el periodista evitará la intromisión gratuita y las especulaciones innecesarias sobre sus sentimientos y circunstancias».

En la revisión de la documentación de los nueve Colegios Profesionales de Periodistas creados en España disponible online solo en tres (Andalucía, Cataluña y Galicia) se encuentran muestras de interés en orientar a los comunicadores en la temática del suicidio.

El Colexio Profesional de Xornalistas de Galicia trabajó con la Xunta de Galicia en la elaboración del Plan de Prevención del Suicidio integrado por una guía de estilo para el tratamiento informativo de esta cuestión, talleres para periodistas e iniciativas para elaborar protocolos e instrumentos de coordinación de los medios con otros sistemas, como el sanitario o el social. La guía desarrollada por esta entidad es bastante práctica pues ofrece una breve y concisa lista de pautas para tratar el suicidio en la línea de las recomendaciones de la OMS que desde el principio con una actitud proactiva: "habla sobre las muertes por suicidio, pero también sobre los factores de riesgo y los medios de prevención».

El Col-legi de Periodistes de Catalunya incluye directamente en su código ético un apartado específico para el suicidio en el que prioriza el respeto y la dignidad de las personas por encima de cualquier información y defiende que «los casos de suicidio sólo se difundirán cuando tengan relevancia personal o sean de manifiesto interés público, teniendo en cuenta, además, el riesgo de un efecto mimético».

El Colegio de Periodistas de Andalucía celebró en colaboración con la Junta de Andalucía en el año 2015 unas Jornadas de formación sobre comunicación y salud que contaron con un apartado específico para el suicidio y del que se extrajeron una serie de recomendaciones que actualmente ofrecen en su página web. Además, el Colegio andaluz ha actuado en varios casos de noticias en los que consideró que no se había hecho un buen tratamiento informativo con respecto a este tema. 
TABLA 3

Análisis de los Colegios Profesionales de Periodistas de España

\begin{tabular}{|l|l|}
\hline \multicolumn{1}{|c|}{ Organización } & \multicolumn{1}{|c|}{ Publica pautas para el tratamiento del suicidio } \\
\hline FAPE & $\begin{array}{l}\text { No, pero sí sobre «informaciones en las que me- } \\
\text { dien elementos de dolor» }\end{array}$ \\
\hline Colegio Profesional de Periodistas de Asturias & No. \\
\hline Colegio Profesional de Periodistas de la Rioja & No. \\
\hline $\begin{array}{l}\text { Colegio Profesional de Periodistas de Castilla y } \\
\text { León }\end{array}$ & No. \\
\hline Colegio de Periodistas Región de Murcia & No. \\
\hline Colexio Profesional de Xornalistas de Galicia & Ś, ofrece una guía para los periodistas. \\
\hline Colegio Profesional de Periodistas de Andalucía & Ś, incluye un apartado en su código ético. \\
\hline Colegio Vasco de Periodistas & No. \\
\hline Colegio de Periodistas de Navarra & No. \\
\hline Col·legi de Periodistes de Catalunya & Sí, ofrece una guía para los periodistas. \\
\hline
\end{tabular}

Fuente y elaboración propias.

Posteriormente se continuó con el análisis de las asociaciones internacionales. En este caso, la cuestión estaba en sí ofrecían algún tipo de información a los periodistas (pautas, noticias que hablasen sobre el tema...). De las 23 asociaciones en ninguna se ofrecía una guía como tal, aunque en el 60,9\% de ellas se encontró algún tipo de noticia que hablase sobre el suicidio o su tratamiento, sin pronunciarse ni dar consejos. En las restantes $(39,1 \%)$ entidades no contenían se detectó ninguna referencia a cómo informar sobre el suicidio.

\subsection{AnÁlisis de nOticias}

Es importante que los medios y asociaciones profesionales promuevan pautas para informar sobre suicidio, pero aún lo es más que las apliquen o que tomen como referencia autoridades en materia de salud. La guideline de la OMS se usa como referencia de análisis sobre un total de 258 noticias de los once medios de referencia que integran la muestra. El primer resultado se desprende del proceso metodológico: sólo uno de cada diez textos sobre suicidio se refería realmente a un acto suicida como eje vertebrador principal de la narración informativa. 
Los resultados del análisis son claros. En ninguno de los textos informativos españoles analizados se informa sobre líneas de ayuda, indicadores de riesgo o alternativas al suicidio. El dato más negativo es que en casi una de cada tres informaciones aún se siguen dando detalles que describen el modo en que se realizó el acto suicida. No aparece ninguna recomendación con 100\% de seguimiento en todos los medios aunque son muy elevados los casos en los que se evitan razones simplistas, identificar culpas y evitar fotos del suicida o del escenario del suicidio (tabla 4).

TABLA 4

Porcentaje de noticias de medios nacionales que cumplen cada recomendación

\begin{tabular}{|l|c|}
\hline \multicolumn{1}{|c|}{ Pauta de la OMS } & $\begin{array}{c}\text { Noticias que cumplen } \\
\text { esta recomendación }\end{array}$ \\
\hline Proporcionar información sobre líneas de ayuda y recursos comunitarios & $0,0 \%$ \\
\hline Proporcionar indicadores de riesgo y señales de advertencia & $0,0 \%$ \\
\hline No publicar fotografias o notas suicidas & $96,3 \%$ \\
\hline No aportar detalles específicos del método empleado. & $66,7 \%$ \\
\hline No dar razones simplistas. & $94,4 \%$ \\
\hline No aportar culpas & $94,4 \%$ \\
\hline No resaltar las alternativas al suicidio & $0,0 \%$ \\
\hline Nota media & $50,5 \%$ \\
\hline
\end{tabular}

Fuente y elaboración propias.

Desglosando los resultados se ven comportamientos muy similares. Sobresale el indicador de La Voz de Galicia y El Periódico sobre la no publicación de fotos o notas de suicidas, y de El País y El Periódico en no incorporar al relato la atribución de culpas. Algo que podría explicar la variación de los datos, especialmente en la pauta sobre la no publicación de notas suicidas y fotografias, es que ignoramos el dato de en cuántos de estos casos se disponía de esta información para poder valorar si fue una decisión de exclusión o simplemente que no se tenía el material gráfico. En segundo lugar, los porcentajes más altos en las pautas sobre no aportar culpas, razones simplistas o detalles específicos, también se podrían deber a que en muchos de los casos analizados no se da una cobertura extensa del tema, por lo que tampoco hay un gran margen para cometer estos errores. 
TABla 5

Porcentaje de noticias de medios nacionales que cumplen cada recomendación según el medio

\begin{tabular}{|l|c|c|c|c|c|}
\hline \multicolumn{1}{|c|}{ Pedios nacionales } \\
\hline \multicolumn{1}{|c|}{ Pauta de la OMS } & El País & El Mundo & Vanguardia & La Voz & Periódico \\
\hline $\begin{array}{l}\text { Informa sobre líneas de ayuda y recur- } \\
\text { sos comunitarios }\end{array}$ & $0,0 \%$ & $0,0 \%$ & $0,0 \%$ & $0,0 \%$ & $0,0 \%$ \\
\hline $\begin{array}{l}\text { Incluye indicadores de riesgo y señales } \\
\text { de advertencia }\end{array}$ & $0,0 \%$ & $0,0 \%$ & $0,0 \%$ & $0,0 \%$ & $0,0 \%$ \\
\hline $\begin{array}{l}\text { Evita fotografias o notas suicidas } \\
\text { Evita detalles específicos del método } \\
\text { empleado. }\end{array}$ & $96,0 \%$ & $96,6 \%$ & $92,9 \%$ & $100,0 \%$ & $100,0 \%$ \\
\hline Evita dar razones simplistas. & $96,0 \%$ & $93,1 \%$ & $92,9 \%$ & $92,3 \%$ & $100,0 \%$ \\
\hline Evita aportar culpas & $100,0 \%$ & $93,1 \%$ & $92,9 \%$ & $76,9 \%$ & $100,0 \%$ \\
\hline No resalta las alternativas al suicidio & $0,0 \%$ & $0,0 \%$ & $0,0 \%$ & $0,0 \%$ & $0,0 \%$ \\
\hline Porcentaje medio & $51,4 \%$ & $50,2 \%$ & $49,4 \%$ & $47,3 \%$ & $53,8 \%$ \\
\hline
\end{tabular}

Fuente y elaboración propias.

La pauta de «no aportar detalles específicos del método empleado» es una de las más vulneradas y esto se debe a la gran carga sensacionalista que se le atribuye a esta temática en los periódicos españoles. Nos encontramos con casos de suicidio como el del banquero Miguel Blesa, en el que todos los medios realizaron una cobertura detallada hasta el punto de describir todos y cada uno de los pasos que dio el presidente de Caja Madrid para llevar a cabo su suicidio con un rifle de caza.

En el gráfico 1 observamos la nota media de cada uno de los medios, siendo 0 un no cumplimiento de ninguna de las recomendaciones en ninguna de las noticias y el 10 un cumplimiento de cada una de las pautas en todos los casos. Podemos ver como en todos los casos se ronda el suficiente, pero destacando que como vimos en la tabla 3 hay un reparto muy heterogéneo del seguimiento de las reglas.

Al aplicar la ficha de análisis con las recomendaciones de la OMS a las noticias publicadas por los medios internacionales de referencia que integran la muestra intencional se observa una situación dispar. The Guardian informa sobre ayudas y recursos comunitarios de apoyo en nueve de cada diez informaciones $(88,2 \%)$ pero el resto ni lo menciona. También The Guardian (35,3\%) junto a The New York Times y The Washington Post son los únicos que incorporan al relato indicadores de riesgo o señales de advertencia. Solo en Corrieri della Sera y Le Monde se respeta siempre la pauta de no publicar fotos o notas suicidas. Los porcentajes de la situación detectada en cada uno de los 7 medios se detallan en la tabla 5 . 
10,00

Nota media

$$
8,00
$$

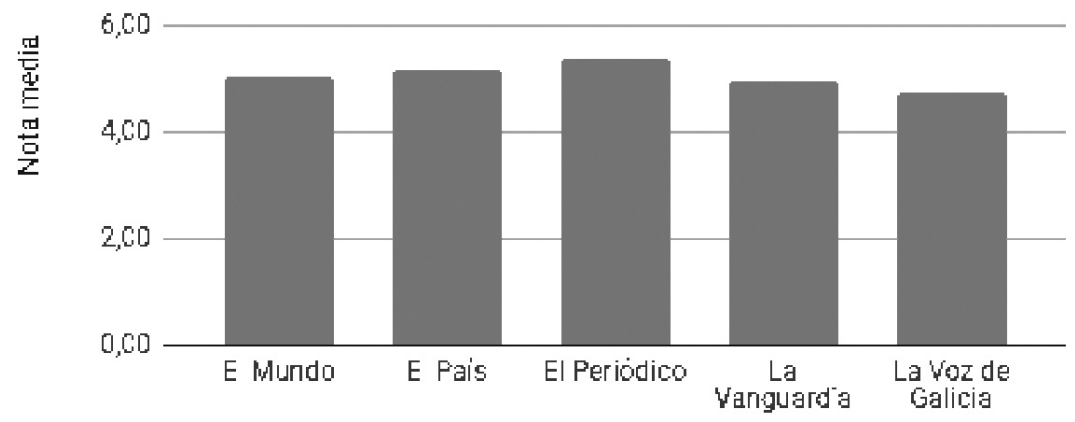

Medos

Fuente y elaboración propias.

\section{GrÁFICO 1}

\section{Notas medias de los medios nacionales}

Para las pautas en las que se consiguen los mayores porcentajes de seguimiento hacemos una observación semejante a la de los medios nacionales: en muchos casos no se dan datos del método empleado porque las informaciones son muy breves o se centran en declaraciones de la policía. Además, en estos apartados encontramos porcentajes muy bajos en relación a los resultados españoles. Es llamativo que el New York Times especifique cómo se llevó a cabo el acto en casi tres de cada cuatro de las piezas analizadas. 
TABLA 6

Porcentaje de noticias de medios internacionales que cumplen cada recomendación según el medio

\begin{tabular}{|c|c|c|c|c|c|c|c|}
\hline \multicolumn{8}{|c|}{ Medios internacionales } \\
\hline Pauta de la OMS & $\begin{array}{c}\text { Corrieri } \\
\text { della Sera }\end{array}$ & El País & Le Monde & $\begin{array}{c}\text { Público } \\
\text { (Portugal) }\end{array}$ & $\begin{array}{l}\text { The } \\
\text { Guardian }\end{array}$ & $\begin{array}{l}\text { The New } \\
\text { York Times }\end{array}$ & $\begin{array}{l}\text { The } \\
\text { Washington } \\
\text { Post }\end{array}$ \\
\hline $\begin{array}{l}\text { Informa sobre líneas } \\
\text { de ayuda y recursos } \\
\text { comunitarios }\end{array}$ & $0,0 \%$ & $0,0 \%$ & $0,0 \%$ & $0,0 \%$ & $88,2 \%$ & $0,0 \%$ & $0,0 \%$ \\
\hline $\begin{array}{l}\text { Incluye indicadores } \\
\text { de riesgo y señales } \\
\text { de advertencia }\end{array}$ & $0,0 \%$ & $0,0 \%$ & $0,0 \%$ & $0,0 \%$ & $35,3 \%$ & $11,1 \%$ & $5,6 \%$ \\
\hline $\begin{array}{l}\text { Evita fotografias o } \\
\text { notas suicidas }\end{array}$ & $100,0 \%$ & $96,0 \%$ & $100,0 \%$ & $88,9 \%$ & $90,2 \%$ & $72,2 \%$ & $86,1 \%$ \\
\hline $\begin{array}{l}\text { Evita detalles es- } \\
\text { pecíficos del mé- } \\
\text { todo empleado. }\end{array}$ & $55,6 \%$ & $64,0 \%$ & $62,5 \%$ & $66,7 \%$ & $74,5 \%$ & $27,8 \%$ & $38,9 \%$ \\
\hline $\begin{array}{l}\text { Evita dar razones } \\
\text { simplistas. }\end{array}$ & $88,9 \%$ & $96,0 \%$ & $87,5 \%$ & $100,0 \%$ & $94,1 \%$ & $72,2 \%$ & $77,8 \%$ \\
\hline Evita aportar culpas & $96,3 \%$ & $96,0 \%$ & $100,0 \%$ & $100,0 \%$ & $64,7 \%$ & $33,3 \%$ & $55,6 \%$ \\
\hline $\begin{array}{l}\text { No resalta las alter- } \\
\text { nativas al suicidio }\end{array}$ & $0,0 \%$ & $0,0 \%$ & $0,0 \%$ & $0,0 \%$ & $0,0 \%$ & $0,0 \%$ & $0,0 \%$ \\
\hline Porcentaje medio & $48,7 \%$ & $50,7 \%$ & $50,0 \%$ & $50,8 \%$ & $63,9 \%$ & $31,7 \%$ & $37,7 \%$ \\
\hline
\end{tabular}

Fuente y elaboración propias.

\section{Conclusiones}

El suicidio es omitido o tratado de forma negligente en gran medida, un hecho que no contribuye a solucionar el problema que supone el alto índice de muertes por esta causa en España y en el resto del mundo. En muchos casos se escoge silenciar este suceso aludiendo al efecto llamada, o efecto Werther. Sin embargo, la OMS nunca recomienda esta práctica, porque de igual manera que en los casos de violencia de género, los suicidios necesitan una atención especial. Además, las conductas más preocupantes son aquellas en las que se llega a culpar a la propia persona de la enfermedad mental o se achacan razones simplistas a su muerte. Cabe destacar que relacionado en el incorrecto tratamiento de este tipo de informaciones está el estigma que rodea tanto la cuestión como a la salud mental en general. 
Por otra parte, los resultados globales del análisis muestran que gran parte de los medios alcanzan un 50\% de seguimiento de las recomendaciones de la OMS. Pero al observar los resultados nos damos cuenta de su heterogeneidad. No hay patrones diferenciales en función del territorio y los comportamientos son dispares tanto en medios españoles, como en los europeos o los americanos.

La muestra integrada por diarios generalistas españoles con mayor audiencia no permite hablar de una atención preferencial al abordaje informativo del suicidio en los libros de estilo o en los códigos deontológicos. De los cinco analizados, solo se encontraron referencias concretas en dos y solo uno de ellos en línea con las recomendaciones de la OMS. En el caso de los medios internacionales solo en uno, The Guardian, se encontraron indicaciones específicas sobre el tema.

Tampoco se observa una gran preocupación en las organizaciones profesionales, a pesar de que se trata de una causa de muerte por encima de otras causas que habitualmente merecen cobertura informativa. No se localizaron recomendaciones específicas en el portal web de la Federación de Asociaciones de la Prensa, ni en seis de los nueve Colegios de Periodistas, ni en las 23 organizaciones profesionales internacionales analizadas.

La autorregulación profesional, en colaboración con expertos del campo de salud es todavía una tarea pendiente en la profesión periodística en España. En Galicia y Andalucía la tarea se hizo en común con las autoridades gubernamentales de Sanidad como primer acercamiento a contar con guías de abordaje integral del suicidio y en Cataluña se incluye en las pautas éticas, pero sin recomendaciones concretas.

El análisis de contenido de las informaciones sobre suicidio en los diarios nacionales e internacionales aún no permite hablar de rutinas asociadas a una cobertura de este tipo de acontecimientos que se aproxime a las recomendaciones de la OMS. El seguimiento de las pautas no comprende solo el informar sobre el hecho como se haría con sucesos similares, sino que también incluye la visibilización de acciones de apoyo y la participación de los medios como agentes divulgadores de textos que redunden en favor de aportar dinámicas o pautas comportamentales que ayuden a la prevención del suicidio de modo proactivo.

Los datos, como se evidencia, responden a las preguntas de investigación al identificar medios y organizaciones que incluyan el tratamiento del suicidio en sus libros de estilo, al señalar las diferencias entre los medios nacionales y los internacionales en su comportamiento ante actos suicidas y al ratificar como en ocasiones aún se recurre a aspectos morbosos al detallar el proceso del suicidio, publicar fotografias o notas e incluso hacer derivaciones de culpabilidad. No es posible sin embargo una diferenciación inequívoca de tendencia de las actitudes mediáticas a alinearse con el efecto Werther o con el efecto Papageno, aunque los indicadores manejados apuntan a 
un predominio de las políticas informativas de silencio deliberado (Werther) por la falta de visibilidad al comparar el número de suicidios con el número de noticias, por referencias claras a evitar estas informaciones para no suscitar comportamientos de imitación y en la notoria ausencia de contenidos que se alineen con actitudes favorables a promover la prevención a través de la incorporación al relato de datos significativos como entidades de apoyo o síntomas de identificación de posibles conductas de riesgo.

\section{Referencias bibliográficas}

Carrascosa, Lara (2015). El tratamiento sensacionalista y amarillista del suicidio por razones económicas en la prensa estatal y canaria a inicios de la crisis actual (agosto 2007-enero 2009). Revista internacional de Historia de la Comunicación, 5, 52-75. ISSN-e 2255-5129. Recuperado de https://dialnet.unirioja.es/ejemplar/424520.

Catalán-Matamoros, D., Axelsson R. y Strid, J. (2007). How do newspapers deal with health in Sweden? A descriptive study. Patient Education and Counseling, 67, 78-83.

Cohen, B.C. (1963). The press and foreign policy. Princeton, NJ: Princeton University Press.

Colegio Profesional de Periodistas de Andalucía (2018). El Colegio profesional de Periodistas expresa su preocupación sobre casos de suicidios en Cádiz. Recuperado de https:// periodistasandalucia.es/2018/01/12/colegio-periodistas-expresa-preocupacion-laspublicaciones-casos-suicidios-cadiz/

Colegio Profesional de Periodistas de Cataluña (2016). Código deontológico. Recuperado de https://fcic.periodistes.cat/es/codi-deontologic/

Colexio Profesional de Xornalistas de Galicia (2017). Recomendacións profesionais sobre información e sufrimento humano. Recuperado de http://www.xornalistas.org/novaseventos/novas/o-cpxg-e-o-copg-presentan-as-recomendacions-profesionais-sobreinformacion-e-sufrimento-humano-/

El Periódico (1993). Estatuto de redacción de El Periódico de Catalunya.

Dumon, E., y Portzky, G. (2014). Directrices de actuación para la Prevención, Intervención y Postvención del suicidio en el entorno escolar. Europa Belgica. Recuperado de http://blogs.grupojoly.com/con-la-venia/files/2015/06/HerramientaMedios Comunicacion.pdf

Garrido-Fabián, F, Eleazar Serrano-López A. y Catalán-Matamoros, D. (2018). El uso por los periodistas de las recomendaciones de la OMS para la prevención del suicidio. El caso del periódico Abc. Revista Latina de Comunicación Social. Recuperado de http:// www.revistalatinacs.org/073paper/1283/42es.html DOI: 10.4185/RLCS-2018-1283

Goulden, R., Corker E., Evans-Lacko, S., Rose, D., Thornicroft, G. y Henderson, C. (2011) Newspaper coverage of mental illness in the UK, 1992-2008. BMC Public Health, 11, 796. 
Herrera Ramírez, R., Ures Villar, M.B., y Martínez Jambrina, J.J. (2015). El tratamiento del suicidio en la prensa española: ¿efecto werther o efecto papageno?. Revista de la Asociación Española de Neuropsiquiatría, 35(125), 123-134. Recuperado de http://dx.doi. org/10.4321/S0211-57352015000100009

Instituto Nacional de Estadística (2016). Defunciones por suicidios en 2016. Cifras INE. Recuperado de http://www.ine.es/jaxiT3/Tabla.htm?t=7947

Instituto Nacional de Estadística (2016). Causas múltiples de defunción en 2016. Cifras INE. Recuperado de http://www.ine.es/jaxiT3/Tabla.htm?t=7947

Ladwig, K.H., Kunrath, S., Lukaschek, K., \& Baumert, J. (2012). The railway suicide death of a famous German football player: impact on the subsequent frequency of railway suicide acts in Germany. Journal of affective disorders, 136(1-2), 194-198.

Littmann, S.K. (1985). Suicide epidemics and newspaper reporting. Suicide and Life-Threatening Behavior, 15(1), 43-50.

Llobet, L. (2006). ¿La función social del periodismo o periodismo social? Unirevista, 1-11.

López, A.O., y Fernández, D.G. (2015). Suicidio y libertad de información: entre la relevancia pública y la responsabilidad. Revista de Comunicación de la SEECI, 38, 70-114.

Los medios de comunicación tienen que gritar que el suicidio no es una vergüenza, no tiene ninguna connotación moral negativa. (2016). Recuperado de http://fape.es/losmedios-de-comunicacion-tienen-que-gritar-que-el-suicidio-no-es-una-verguenza-notiene-ninguna-connotacion-moral-negativa/

McCombs, M.E., y Shaw, D.L. (1972). The agenda-setting function of mass media. Public opinion quarterly, 36(2), 176-187.

Morán, A. (2015). 800.000: el suicidio, el mal invisible [Documental]. España: 93 Metros.

Müller, F. (2011). El «Efecto Werther»: gestión de la información del suicidio por la prensa española en el caso de Antonio Flores y su repercusión en los receptores. Cuadernos de Gestión de Información, 1, 65-71. Recuperado de http://revistas.um.es/gesinfo/article/ view/207541

Muñoz Chávez, L.F. (2014) Caracterización de noticias sobre suicidio en medios impresos en Colombia Tesis presentada para optar al título de: Especialista en Psiquiatría Universidad Nacional de Colombia Facultad de Medicina, Departamento de Psiquiatría Bogotá. Recuperado de bdigital.unal.edu.co/45820/1/05599223.2014.pdf

Niederkrotenthaler, T., Voracek, M., Herberth, A., Till, B., Strauss, M., Etzersdorfer, E., y Sonneck, G. (2010). Role of media reports in completed and prevented suicide: Werther v. Papageno effects. The British Journal of Psychiatry, 197(3), 234-243.

Norris, B., Jempson, M., Bygrave, L., Thorsen, E. (2001). Reporting suicide worldwide. Media responsabilities. Recuperado de http://www.mediawise.org.uk

Olmo López, A. y García Fernández, D. (2015). Suicidio y libertad de información: entre la relevancia pública y la responsabilidad. Revista de Comunicación de la SEECI (Sociedad Española de Estudios de la Comunicación Iberoamericana), 38. Doi:http://dx.doi. org/10.15198/seeci.2015.38.70-114 
OMS/Organización Mundial de la Salud (2000). Prevención del suicidio. Un instrumento para profesionales de los medios de comunicación. Recuperado de http://www.who. int/mental_health/publications/suicide_prevention/es/index. htm

País, E. (1991). Manual de estilo. Madrid: Prisa.

Phillips, D.P., Lesyna, K., \& Paight, D.J. (1992). Suicide and the media. Assessment and prediction of suicide. New York, NY, US: Guilford Press, 499-519.

Phillips, D.P. (1985). The Werther Effect. The Sciences, 25, 33-39.

Philips, D.P. (1982). The impact of fictional television stories on US adult fatalities: new evidence on the effect of the mass media on violence. American journal of sociolog $\gamma, 87$, 1340-1359.

Rativa, J.S., Ruiz, V.A. y Medina-Pérez O.A. (2013). Análisis de las noticias sobre suicidio publicadas en un diario local de Quindío, Colombia, entre 2004 y 2011. Rev. Cubana Hig. Epidemiol, 51, 184-191.

Rubiano, H., Quintero, T. y Bonilla, C. I. (2007). El suicidio: más que una noticia, un tema de responsabilidad social. Palabra clave, 10, 2.

Sánchez Girón, J. (2016). El tratamiento del suicidio en la prensa digital española según las recomendaciones de la Organización Mundial de la Salud (OMS). Valladolid: Universidad de Valladolid.

Scherr, S. y Steinleitner, A. (2015). Zwischen dem Werther- und Papageno-Effekt. Nervenarzt, 86, 557-565.

de la Serna, V. (Ed.). (1996). El Mundo, libro de estilo. Madrid: Temas de hoy.

Sisask, M. \& Värnik, A. (2012). Media Roles in Suicide Prevention: A Systematic Review. Int. J. Environ. Res. Public Health, 9 , 123-138. Doi: http://dx.doi.org/10.3390/ ijerph9010123

Steven, S. (2003). Media coverage as a risk factor in suicide. Journal Epidemiology Community Health, 57(4), 238-240.

Thornicroft, A., Goulden, R., Shefer, G., Rhydderch, D., Rose, D., Williams, P., Henderson, C. et al. (2013). Newspaper coverage of mental illness in England 2008-2011. The British journal of psychiatry, 202(55), 64-69.

Urzúa Aracena, Mario (2003). Suicidio, intimidad e información. Documentación de las Ciencias de la información. Chile: Facultad de Comunicación Universidad Católica de Concepción, 26, 351-358.

Voz de Galicia, L. (1992). Manual de estilo. A Coruña: Biblioteca Gallega.

Wasserman, I. (1984). Imitation and suicide: A re-examination of the Werther effect. American Sociological Review, 49, 427-436. 
